\title{
UNA APROXIMACIÓN A LAS REPRESENTACIONES DEL ALUMNADO SOBRE EL UNIVERSO
}

\author{
AFONSO LÓPEZ, R. ${ }^{1}$, BAZO GONZÁLEZ, C. ${ }^{2}$, LÓPEZ HERNÁNDEZ, M. ${ }^{3}$, \\ MACAU FÁBREGA, M.D. y RODRÍGUEZ PALMERO, M.L. ${ }^{s}$ \\ ${ }_{1}$ IB Tomás de Iriarte. Santa Cruz de Tenerife. \\ IB El Chapatal. Santa Cruz de Tenerife. \\ 3 IB Mencey Acaymo. Güimar. Santa Cruz de Tenerife. \\ - Inspección de Enseñanza Secundaria. Consejería de Educación. Gobierno de Canarias. \\ 5 IB Dr. A. González y González. Tejina. Santa Cruz de Tenerife. \\ Grupo Anambro. Centro de profesores de La Laguna \\ C/ La Isa, $n^{\mathbb{2} 33}$. Urbanización Cercado Mesa. La Laguna. Santa Cruz de Tenerife.
}

\section{SUMMARY}

This study explains how the children from secondary school (age 14-18) represent the structure of the universe and the way it may be useful in daily teaching.

The results obtained describe the same conceptualization difficulties that the younger students already have, as well as the need for studying deeply the process for learning of those students as far as, having dealt with those contents before, they have not reached the expected scientific knowledge concerning the topic.

\section{INTRODUCCIÓN}

El firmamento, sea en una luminosa mañana de sol o en una noche repleta de estrellas, viene fascinando a la humanidad desde el instante en el que inicia su andadura en este planeta. El Universo, su forma, su tamaño, sus componentes, su origen y su evolución son temas que, del mismo modo, atraen al alumnado de todos los niveles educativos, desde la enseñanza primaria hasta la universitaria. En definitiva, la cuestión que a lo largo de la historia ha centrado la investigación en este tema es: ¿Cómo es y cómo funciona el Universo? «Una pregunta tan simplemente formulada como ésta mueve a observadores, investigadores, estudiantes y docentes; y en el intento de darle respuesta todos están haciendo ciencia, todos están construyendo un conocimiento científico compartido» (Rodríguez Palmero y Rodríguez Palmero, 1995).

La comprensión del sistema Tierra-Sol-Luna representa, pues, uno de los elementos clave, si no el fundamental, en la historia y en la evolución de las ideas $y$, consecuentemente, en el desarrollo científico. Como plantean Jones, Lynch y Reesink (1987): «La necesidad del modelo heliocéntrico opuesto a la descripción de la Tierra como centro del sistema solar tiene un enorme significado intelectual. La transición representa un indicador clave de las tensiones que existen entre mito, magia, ciencia y religión». Estos cuatro aspectos ban ejercido una influencia crucial en la evolución de las ideas relativas al Universo mostrando, quizás de manera más evidente que en otros campos científicos, la idea ya comentada de construcción progresiva del conocimiento que caracteriza la ciencia. Y, en la medida en que de este modo se ha producido el conocimiento científico a lo latgo de la historia, los procesos de enseñanza/aprendizaje en los estudiantes deben guiarse por la misma premisa de apropiación progresiva de dicho conocimiento de manera que formen parte de sus estructuras cognitivas. Esto no es posible con planteamientos docentes acumulativos; en este sentido, y en este campo concreto, resulta especialmente significativo el siguiente comentario que Piaget ha expresado en uno de sus artículos: 
«En el campo del pensamiento toda la historia de la ciencia, desde el geocentrismo a la revolución copernicana, desde las falsedades absolutas de la física aristotélica a la relatividad de los principios de inercia de Galîleo y la teoría de la relatividad de Einstein, muestra que ha lievado siglos liberarnos de los errores sistemáticos, de las ilusiones causadas por el punto de vista inmediato oponiéndose al pensamiento sistemático «descentrado $m[\ldots]$

„Para la ciencia cambiar de una perspectiva geocéntrica a otra heliocéntrica requirió una gigantesca hazaña de descentrismo. Pero el mismo tipo de procesos puede verse en Ios niños...» (Piaget, citado por Nussbaum, 1979).

Resulta especialmente paradójico que un campo tan potencialmente educativo en relación con el conocimiento científico como es el del Universo haya ido desapareciendo de los programas de Geología de COU de diferentes distritos universitarios y se haya visto reducido en los contenidos seleccionados para BUP y para ESO. Una explicación razonable plantean Jones, Lynch y Reesink (1987); estos autores exponen que: «La astronomía era considerada como el más importante componente del currículo de ciencias de secundaria del siglo XIX y principios deI XX [...] El auge de la enseñanza de la ciencia heurística con su énfasis en la experiencia directa es probablemente la principal razón de su subsiguiente defunción».

El estudio del Universo ofrece, desde nuestro punto de vista, una posibilidad única de que los estudiantes aprendan a interpretar la realidad que los rodea, construyan unos conocimientos útiles para resolver problemas que surjan incluso en la vida cotidiana. De hecho, son abundantes los trabajos publicados relativos a la comprensión del alumnado, generalmente en enseñanza primaria, que muestran de qué manera se utilizan no sólo los conceptos estrictamente astronómicos sino también muchos otros relacionados con la física o las matemáticas y que son de uso cotidiano (Nussbaum y Novak, 1976; Nussbaum, 1979; Klein, 1982, Sneider y Pulos, 1983; y Jones, Lynch y Reesink,1987). En ellos se plantea la comprensión infantil de la Tierra como cuerpo cósmico, de la relación entre la Tierra y el Sol, de las distintas explicaciones o nociones de día y noche, de la forma de la Tierra y la gravedad, de las relaciones que se establecen en el sistema Tierra-Sol-Luna, etc. Estas investigaciones han puesto de manifiesto serias dificultades de aprendizaje en estudiantes de enseñanza primaria que, como señala Klein (1982) evidencian que no se ha desarrollado el entendimiento de la Tierra en el espacio, de su perspectiva, de su rotación, etc., del mismo modo, se pone de manifiesto que una búsqueda de jerarquización de este conocimiento por parte del alumnado en sus procesos de aprendizaje del sistema Tierra-Sol-Luna iguala el desarrollo histórico de las explicaciones científicas (Jones, Lynch y Reesink, 1987).

Partiendo de la teoría del aprendizaje significativo como marco de referencia de nuestra labor docente, hemos asumido la importancia que tienen las concepciones del alumnado en los procesos de enseñanza y de aprendizaje (Osborne y Freiberg, 1990). Como se recordará, el papel de la escuela, desde nuestra perspectiva, se centra en una negociación de significados entre los estudiantes, que tienen su interpretación de la realidad, y el profesorado, que ofrece el conocimiento científicamente producido, de manera que aquéllos puedan aprehenderlo. En este sentido, nuestro interés gira en torno a las relaciones espaciales del sistema Tierra-Sol-Luna así como, fundamentalmente, de este sistema en relación con el resto del Universo; es decir, en el estudio o análisis de los elementos que los estudiantes incorporan, además del sistema ya citado, a la estructura del Universo en su conjunto, o a los modelos globales que la explican. El estudio del sistema Tierra-Sol-Luna en este contexto no debe hacer olvidar su relación con las ideas sobre la Tierra relativas a su forma o a su comportamiento; niños pequeños han mostrado tener dificultades con conceptos como distancia, espacio, fuerza, gravedad... en su uso para poder explicar ambos aspectos (Nussbaum y Novak, 1976). ¿Se repetirian estas concepciones en personas adultas? ¿Se puede entender la estructura del Universo o los modelos que la explican sin haber desarrollado o adquirido estos conceptos? ¿Las representaciones del Universo que se manifiestan son o pueden ser consecuencias de problemas de aprendizaje de dichos conceptos?

Desde ta perspectiva expuesta se ha desarrollado una investigación con alumnado de enseñanza secundaria (14-18 años). La finalidad de este trabajo es conocer las concepciones que manifiestan estas personas para elaborar estrategias posteriores de instrucción docente destinadas a la consecución de aprendizajes más significativos y coherentes desde el punto de vista científico. Como se ha comentado, la bibliografía consultada es extensa en trabajos relativos a la ensenanza primaria; pero no se han encontrado referencias a individuos de mayor edad. Esto ha hecho necesario que, antes de plantear la enseñanza y elaborar los materiales adecuados, se pudiera disponer de una mínima información sobre estas concepciones que a priori confirmara la hipótesis de que se reproducirían, en líneas generales, los resultados de los trabajos elaborados en estudiantes pequeños en Ios aspectos en los que hay coincidencia. Los resultados obtenidos hacen suponer que, efectivamente, podrian reproducirse dichas interpretaciones del Universo en personas adultas, incluso sujetas a escolarización, lo que demuestra que no se han producido los procesos de aprendizaje que la misma pretendía.

\section{MÉTODO}

\section{Sujetos}

El presente estudio se ha elaborado sobre un total de 169 estudiantes de $1^{2}$ de BUP y de geología de COU de tres centros diferentes de la isla de Tenerife, durante los cursos escolares 1990-91, 1991-92 y 1992-93. La distribución responde a lo siguiente: 


\begin{tabular}{|l|c|c|c|c|}
\hline NIVEL & $\begin{array}{c}\text { IB TOMÁS } \\
\text { DE IRIARTE }\end{array}$ & $\begin{array}{c}\text { IB MENCEY } \\
\text { ACAYMO }\end{array}$ & $\begin{array}{c}\text { IB A. GLEZ } \\
\text { Y GLEZ. }\end{array}$ & rOTAL \\
\hline $1^{8}$ BUP & 68 & - & & 68 \\
\hline GEOL COU & - & 87 & 14 & 101 \\
\hline TOTAL & 68 & 87 & 14 & 169 \\
\hline
\end{tabular}

Se han elegido estos niveles porque en las programaciones respectivas se habían incluido contenidos relativos al estudio del Universo. Por la experiencia acumulada se habían detectado dificultades de aprendizaje relativas a Ia localización del sistema Tierra-Sol-Luna en el Universo; esto hizo surgir la necesidad de disponer de una información suficiente que permitiera conocer las concepciones del alumnado para poder trabajar con ellas y llegar, en la medida de lo posible, a desarrollar los aprendizajes planificados. Por lo tanto, la selección de niveles y centros no responde más que al único criterio de que las docentes implicadas requerían dicha información para programar y diseñar las unidades didácticas.

Casualmente, pues, se ha dado la circunstancia de que los centros seleccionados responden a características diferenciadas: uno de ellos es urbano-capitalino (IB Tomás de Iriarte) y los otros dos son rurales-comarcales, uno de la zona sur de la isla (IB Mencey Acaymo) y el otro de la zona norte (IB Antonio González y González).

\section{Instrumento}

La información se ha obtenido utilizando como instrumento la realización de un dibujo; la consigna para elaborarlo ha sido que se representara el Universo localizando en él la situación del planeta Tierra. En algunos grupos se ba solicitado, además, que se explicara la idea que sobre el mismo se tenía.

Se ha optado por este instrumento por su sencillez y eficacia a la hora de manejarlo, teniendo en cuenta los fines para los que fue planificado.

\section{Procedimiento}

Los cuestionarios se han pasado, tanto en $1^{2}$ de BUP como en Geología de COU, a principio de curso; en los cursos acadérmicos en los que se ha solicitado esta tarea al alumnado, Ios temas relativos al Universo eran los que iniciaban la programación. Se han desarrollado en horas habituales de clase.

Antes de la realización de la actividad se ha procurado crear el clima adecuado con objeto de que los estudiantes expresaran libremente sus concepciones; en todo momento se insistio en que el resultado de la tarea que realizaban en modo alguno iba a ser objeto de calificación. La actividad ba sido nominal, decisión que se tomó para que los propios estudiantes compararan estas pri- meras representaciones con las que elaborarán el trabajo planificado en la unidad didáctica.

El tiempo destinado a esta actividad inicial ha oscilado entre 20 y 30 minutos.

Para el análisis de los dibujos y representaciones obtenidas se partía a priori de tres categorías; una primera aproximación al material obtenido confirmó la idoneidad de las mismas, pero además hubo que añadir una nueva categoría de difícil definición. Esto hizo que en casos puntuales fueran precisas algunas aclaraciones verbales a través de entrevistas personales.

Los criterios que se han establecido para poder delimitar Ias diferentes categorías son los siguientes:

A. Situación y dimensión del planeta Tierra. Este criterio se refiere a la situación del planeta Tierra con respecto al Universo, considerando una concepción geocéntri$c a$ aquélla en la que nuestro planeta ocupa una posición central. En cuanto a la dimensión, la interpretación se centra en el tamaño que ostenta la Tierra con respecto a los demás astros, así como la utilización de escalas que indiquen la relación entre el tamaño del planeta y el total del Universo.

B. Situación y dimensión del Sol. Se refiere a la localización y tamaño de esta estrella con respecto al resto de los elementos del dibujo, tratándose de una concepción heliocéntrica cuando este astro resulta ser lo más destacado del esquema.

C. Unidades fundamentales del Universo. Los diferentes astros pueden haberse agrupado formando unidades que se repiten en el dibujo como, por ejemplo, galaxias, sistemas estelares, estrellas y planetas. Los esquemas pueden incluir un mismo tipo de unidad o contener un conjunto plural de las mismas. Se estaría representando, por lo tanto, una concepción acéntrica del Universo.

Atendiendo a estos criterios se han establecido cuatro categorías:

1. Universo «saco».

2. Universo geocéntrico.

3. Universo heliocéntrico.

4. Universo acéntrico.

\section{RESULTADOS}

\section{Universo «saco» (representaciones dudosas)}

Dentro de la primera de las categorías definidas se han incluido algunos dibujos que han resultado de dudosa catalogación, como ya hemos comentado. El porcentaje de estas representaciones que no se corresponden con las categorías siguientes es bajo $(4,14 \%)$. Se destaca en este apartado lo que hemos identificado como una consideración de un Universo "saco" en el que se incluyen dife- 


\section{Figura 1}

Algunos de los dibujos que reflejan otras representaciones.

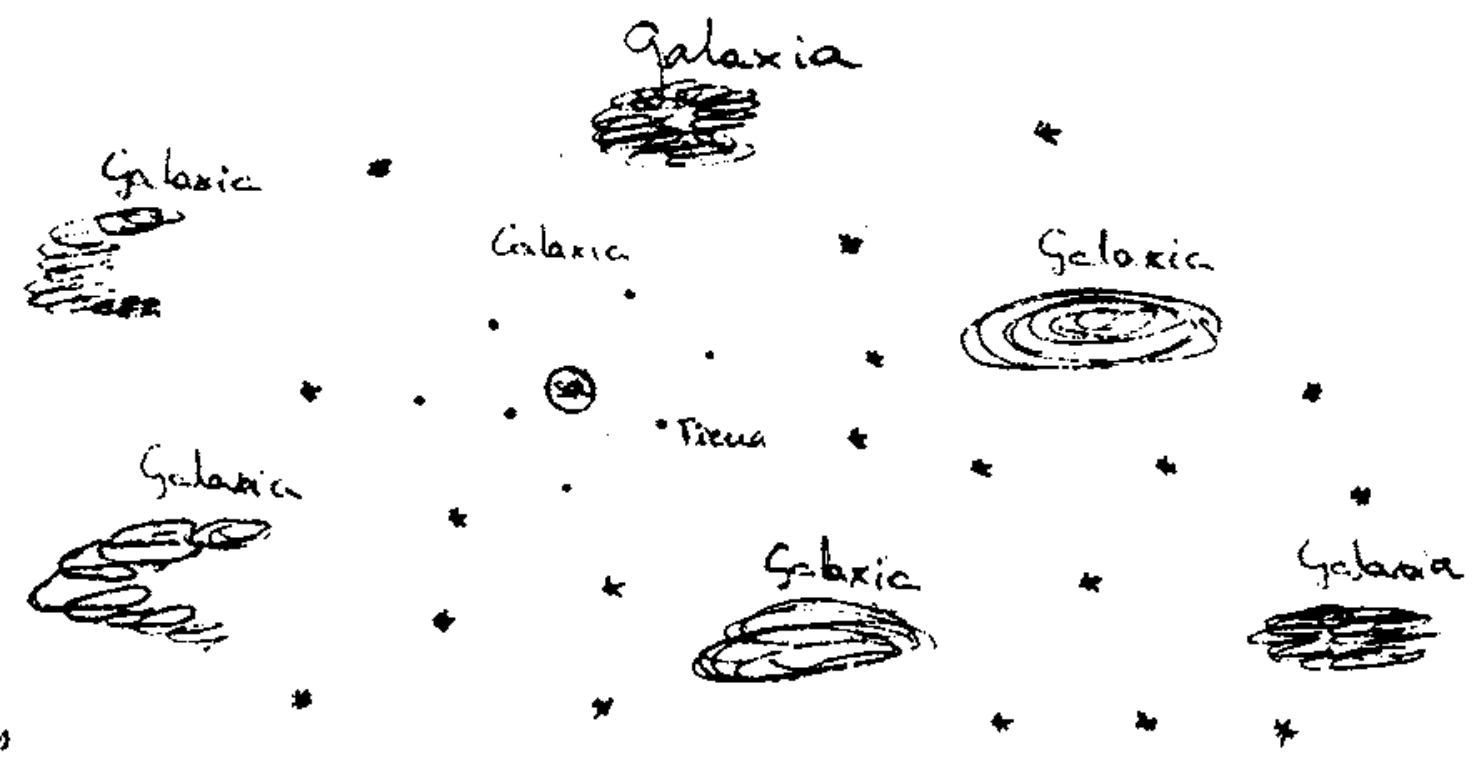

- plaustas del sisturex Sar

c. 0.0 .

I.B. Mewrey Acaymo

C.O.U. I.B. Mencer Acaymo.

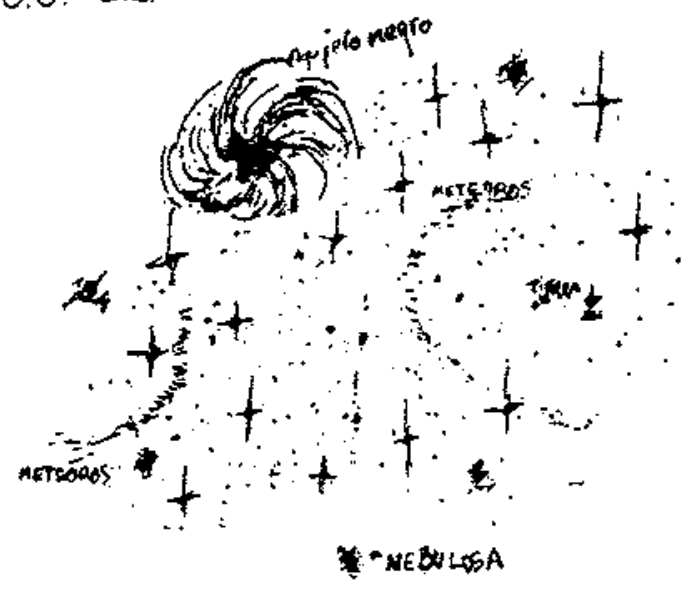


rentes elementos sin delimitar su estructura. También forman parte de este grupo algunos dibujos en los que se representan una serie de puntos y círculos indicados como planetas, estrellas, sistemas estelares, nebulosas, etc. y donde se señala la posición de la Tierra. En la figura 1 se han elegido algunos ejemplos de estas representaciones.

Figura 2

Esquemas más significativos sobre la concepción geocéntrica.
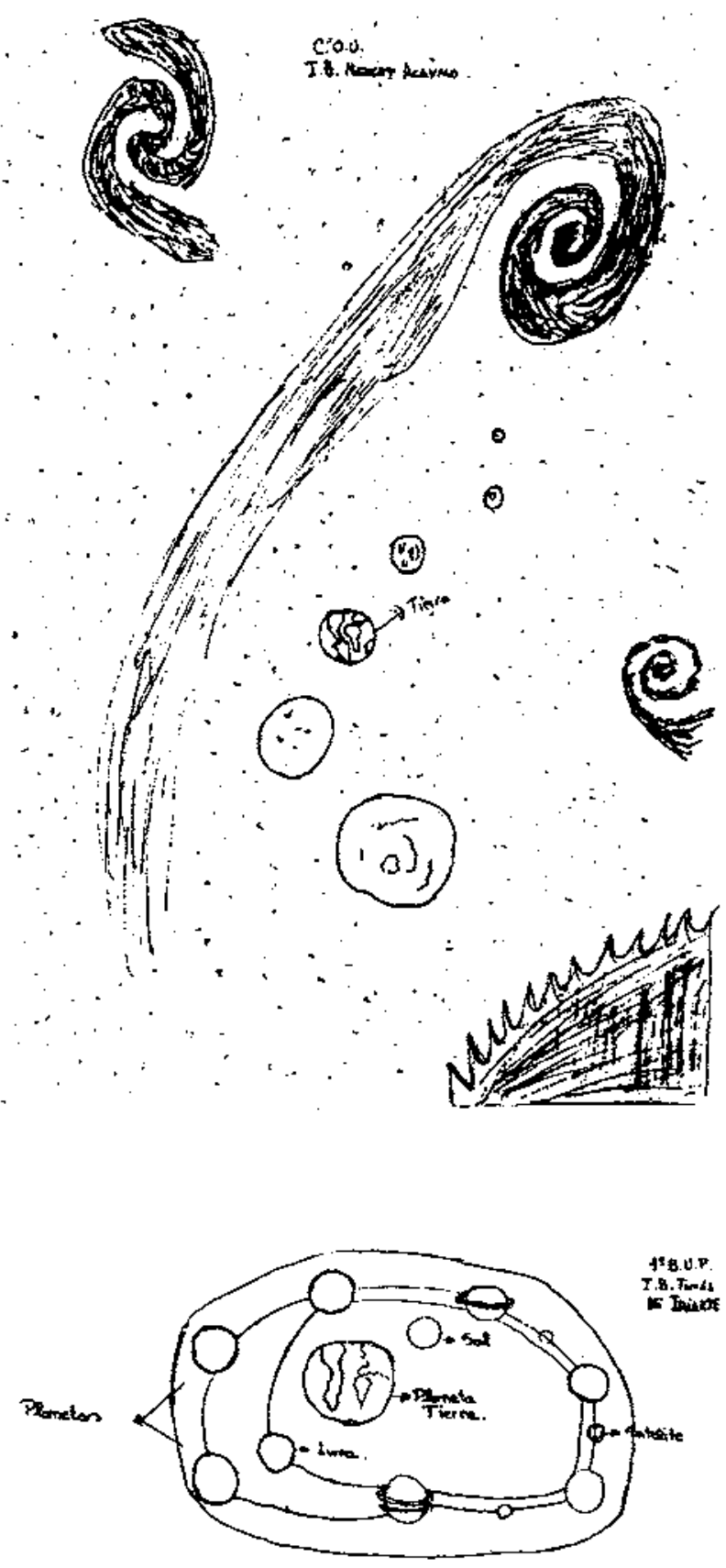

\section{Concepción geocéntrica}

La Tierra está situada en una posición destacada rodeada por el resto de los astros, que a mentdo giran alrededor en órbitas circulares o elípticas, para un $9,46 \%$ del total del alumnado (16 personas).

Las concepciones geocéntricas más infantiles incluyen naves espaciales, satélites artificiales, extraterrestres, etc., lo que muestra, también, una clara influencia de los medios de comunicación. Una selección de los dibujos incluidos en esta categoría se expone en la figura 2 .

\section{Concepción heliocéntrica}

El Sol es, para el mayor número de estudiantes, 103, el astro en torno al cual giran los planetas (Ia Tierra entre ellos), en general en órbitas elípticas o circulares, lo que se corresponde con un $60,94 \%$ del total del alumnado.

A menudo se observa en la misma órbita la presencia de dos o más planetas. Esta concepción se representa, en ocasiones, sin dibujar las órbitas de los planetas, colocando éstos a diferentes distancias del Sol.

En algunos de los dibujos se advierte que, rodeando el sistema solar, se encuentran una serie de estrellas (a veces agrupadas en constelaciones), meteoritos, cometas, agujeros negros y planetas. Esos dibujos responden a una concepción más amplia que la referida al sistema solar, pero sin establecer unidades de organización superiores; sigue destacando en ellos la posición central del Sol, por lo que se han incluido en esta categoría. En la figura 3 se pueden observar ejemplos de algunos de estos dibujos.

\section{Concepción acéntrica}

Las unidades esquematizadas son las galaxias en sus tipos fundamentales: espiral, elíptico e irregular. El número total de estudiantes que muestra esta concepción es 43 , lo que supone un $25,44 \%$ del total de personas sondeadas.

En ocasiones el espacio entre las galaxias es rellenado con estrellas, agujeros negros, cometas y planetas.

Con frecuencia se emplean escalas para indicar la situación del sistema solar y de la Tierra con respecto a la totalidad del Universo.

Un caso especial dentro de esta concepción es el de aquellos estudiantes que comprenden que nuestro sistema solar no debe ser el único que podría existir en el Universo; por eso dibujan un conjunto de sistemas esteIares, parecidos al nuestro, designando a uno de ellos como Vía Láctea, lo que, en principio, muestra una confusión entre sistema estelar y galaxia. La figura 4 refleja algunos de los dibujos que se han incluido en esta categoria. 
Figura 3

Dibujos que representan la concepción heliocéntrica.
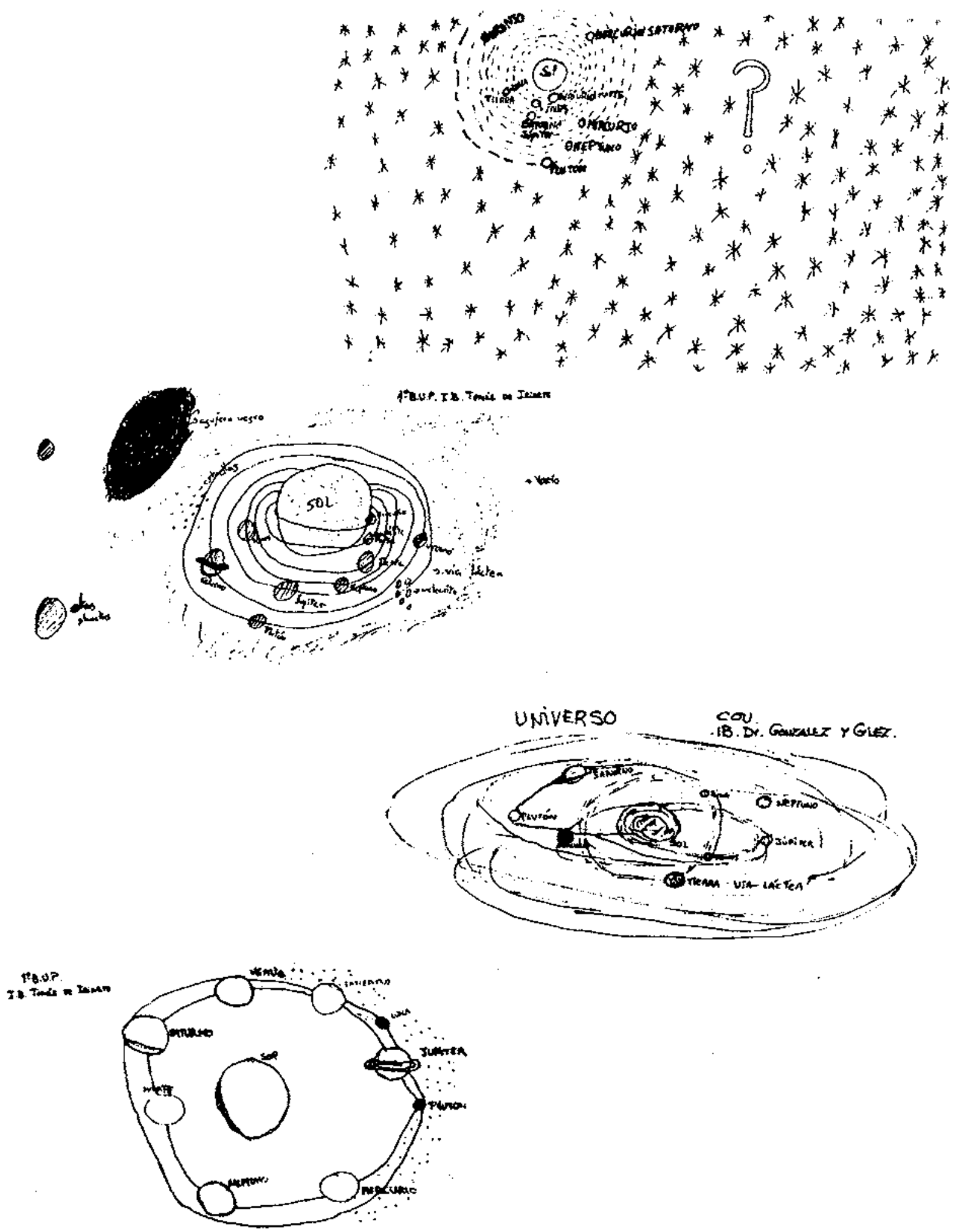
Figura 4

Esquemas que representan ta concepción acéntrica.

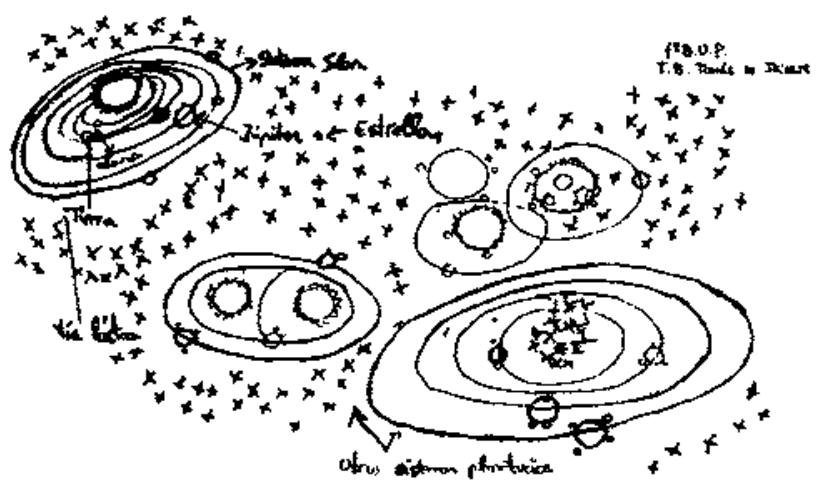

Los resultados obtenidos se muestran en las tablas 1 y II.

Tabla I

Resultados totales absolutos y en porcentajes.

\begin{tabular}{|l|c|c|c|c|}
\hline & $\begin{array}{c}\text { IB } \\
\text { TOMÁS DE } \\
\text { IRIARTE } \\
\text { (12 BUP) }\end{array}$ & $\begin{array}{c}\text { IB } \\
\text { MENCEY } \\
\text { ACAYMO } \\
\text { (COU) }\end{array}$ & $\begin{array}{c}\text { IB DR } \\
\text { A. GLEZ } \\
\text { GLEZ } \\
\text { (COU) }\end{array}$ & TOTAL \\
\hline OTRAS & & $6 / 3,55 \%$ & $1 / 0,59 \%$ & $7 / 4,14 \%$ \\
\hline GEOCÉNTRICA & $3 / 1,77 \%$ & $12 / 7,10 \%$ & $1 / 0,59 \%$ & $16 / 9,46 \%$ \\
\hline HELIOCÉNTRICA & $52 / 30,76 \%$ & $42 / 24,85 \%$ & $9 / 5,32 \%$ & $103 / 60,94 \%$ \\
\hline ACÉNTRICA & $8 / 4,73 \%$ & $32 / 18,93 \%$ & $3 / 1,77 \%$ & $43 / 25,44 \%$ \\
\hline
\end{tabular}

Tabla II

Porcentajes de las distintas concepciones por niveles.

\begin{tabular}{|c|c|c|c|c|}
\hline & OTRAS & GEOCENTRICA & HELOCÉNTRICA & ACÉNTRICA \\
\hline I\% BUP & & $4,76 \%$ & $82,5 \%$ & $12,6 \% \%$ \\
\hline COV & $6,6 \%$ & $12,26 \%$ & $48,11 \%$ & $33,01 \%$ \\
\hline
\end{tabular}

El análisis que se realiza de estos datos es el siguiente:

La concepción mayoritaria es la heliocéntrica $(60,94 \%)$; Ia concepción acéntrica estaría en el segundo lugar $(25,44 \%)$, siendo minoritarias en estos estudiantes las concepciones geocéntrica $(9,46 \%)$ y las otras representaciones $(4,14 \%)$.

Se observa una clara diferencia entre los resultados obtenidos para $1^{2}$ de BUP y para COU. En $1^{2}$ de BUP, el

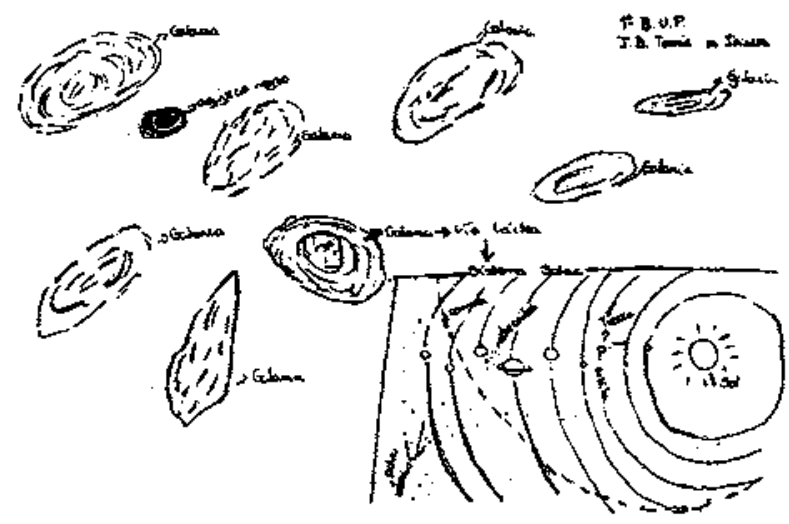

$82,5 \%$ del alumnado manifiesta ideas heliocéntricas frente a una minoría de personas geocéntricas $(4,76 \%)$ y acéntricas $(12,69 \%)$. Sin embargo, en COU los resultados están mucho más repartidos: sigue siendo mayoritaria la concepción heliocéntrica $(48,11 \%)$, pero la acéntrica representa el $33,01 \%$. Es en este nivel donde destaca el elevado porcentaje de representaciones geocéntricas $(12,26 \%)$ y la aparición de otras ideas sobre el Universo $(6,6 \%)$.

No se han detectado diferencias entre los estudiantes de los distintos centros de COU. Tampoco se han detectado diferencias en los resultados obtenidos en los diferentes cursos académicos.

\section{DISCUSIÓN Y CONCLUSIONES}

Es probable que la concepción o idea del Universo como «lo que se ve en una noche clara", como "saco", es decir, como un conjunto o mezcla de "cosas que estấn arriba" represente un problema de conceptualización de la idea de espacio. Cabe la posibilidad de que sean interpretaciones en las que no se tenga una idea clara de la Tierra como marco de referencia y, por lo tanto, de que no se jerarquice adecuadamente que esa Tierra tiene un espacio ilimitado a su alrededor, ocupado por diferentes cuerpos que se estructuran según diferentes sistemas, galaxias, etc. Pueden suponer, además, concepciones que imposibiliten o dificulten la conceptualización de ideas de orden superior como, por ejemplo, la de los propios sistemas estelares. Estas imágenes pueden corresponderse con las representaciones menos elaboradas o más primitivas encontradas por Nussbaum y Novak (1976), Nussbaum (1979), Klein (1982), Sneider y Pulos (1983) y Jones, Lynch y Reesink (1987). Era de esperar que su frecuencia fuera menor en estudiantes de mayor edad (enseñanza secundaria). 
Del mismo modo que hay evidencias de que el concepto de la Tierra se desarrolla con la edad de los individuos, por una serie de aproximaciones sucesivas desde el modelo más egocéntrico hasta modelos científicamente compatibles (Nussbaum, 1979), a medida que se produce un mayor desarrollo psicoevolutivo se van incorporando a las estructuras cognitivas de los estudiantes sistemas explicativos del Universo cada vez más complejos. Las concepciones acéntricas son, consecuentemente, más frecuentes en COU que en $1^{2}$ de BUP, curso en el que su porcentaje es bastante bajo; sin embargo, esta concepción está mucho más representada que la concepción geocéntrica, que ha resultado ser mayoritaria en trabajos relativos al alumnado de enseñanza primaria.

La concepción mayoritaria, tanto en $1^{2}$ de BUP como en Geología de COU, es la heliocéntrica, apareciendo en este último nivel un alto porcentaje de concepciones acéntricas, cono cabría de esperar, por un mayor desarrollo del pensamiento abstracto (estos estudiantes tienen 17-18 años). Cabe, sin embargo, hacer una reflexión, ya que los problemas que se derivan de una mala conceptualización del espacio, y de lo que la Tierra representa en el mismo, se manifiestan en las concepciones que a posteriori se tienen del Universo. «El desarroIlo de determinadas capacidades cognitivas generales aparejado a la edad no significa que haya una mayor adquisición de estos conceptos» (Sneider y Pulos, 1983). De hecho, y puede considerarse un buen ejemplo de lo comentado, es llamativo el elevado número de personas de COU que mantienen una concepción geocéntrica, siendo, incluso, mayor el porcentaje de estudiantes que muestra esta concepción que en $1^{2}$ de BUP.

Como se ve, no se produce un aprendizaje por «salto conceptual» (Jones, Lynch y Reesink, 1987), no se da una sustitución inmediata o automática de un concepto o idea por otro (Moreira, 1994), sino que hay una progresiva diferenciación del concepto de Universo, como demuestran los resultados comparativos de $1^{\mathbb{P}}$ de BUP y COU. Sin embargo, resulta contradictorio encontrar tun mayor porcentaje de concepciones geocéntricas en $\mathrm{COU}$ que en $1^{2}$ de BUP o la ausencia de representaciones de lo que hemos llamado categoría 1 en el nivel de $1^{\circ}$. Cabe la interpretación de que esta categoría deba ser considerada como la tercera y no la primera en el sentido de que se muestran distintos elementos (e incluso se nombran en algunos casos), pero lo que falta es darle a los mismos una organización, una estructura o jerarquía. De hecho, si consideramos el modelo geocéntrico como el más egocentrista (más primitivo en el desarrollo de los ninos), por una parte, esta categoría no respondería a dicho egocentrismo y, por otra, se incorporan más elementos que en dibujos de representaciones incluso heliocéntricas.

Los resultados obtenidos muestran que los intentos de explicación del alumnado, los modelos que se han representado, coinciden con el desarrollo histórico de las explicaciones que la ciencia ba ido dando a lo largo de la historia (Jones, Lynch y Reesink, 1987). De la misma forma que las ideas del Universo han ido cambiando a medida que se incorporaban nuevos enfoques, se aportaban nuevos datos, se definían nuevos modelos y se utilizaban nuevas tecnologías, es previsible que, si estos aspectos son incorporados al aula, cambien las concepciones, los modelos explicativos de nuestros estudiantes. De hecho, se observa que estas ideas van desde las más simples (sea un Universo «saco» o el geocentrismo) hasta modelos más elaborados y abstractos, como la concepción acéntrica, pasando por una imagen heliocéntrica. La construcción de este conocimiento ha supuesto, como ya se ha expuesto, una revolución científica de primera magnitud en la historia de la Ciencia y es, precisamente, esta historia una herramienta muy útil para que el alumnado genere estos aprendizajes de manera no traumática.

En la medida en que el aprendizaje se produce si la persona asimila una nueva información en una estructura cognitiva ya existente (Ausubel, Novak y Hanesian, 1983), conocer esta estructura es fundamental en nuestro trabajo como docentes. Los resultados muestran que, a pesar de haberse trabajado los contenidos comentados en cursos anteriores, no se han producido Ios aprendizajes esperados. Una de las posibles causas es, como concluyen Sneider y Pulos (1983), el uso de un lenguaje que los estudiantes no entienden. Se observa, incluso, que se ha producido distorsión de la información recibida; esta situación, que es previsible que se dé en diferentes lugares y contextos y con distintos estudiantes, debe ser el elemento de partida para diseñar y planificar estrategias que potencien la negociación de significados más coherentes desde el punto de vista científico que resulten eficaces y perdurables en la formación de nuestros estudiantes.

La finalidad del trabajo ha sido conocer las representaciones sobre la estructura del Universo con objeto de preparar una estrategia didáctica. En este sentido, no se han considerado sino aquellos aspectos que babian resultado más conflictivos en su aprendizaje desde la perspectiva de una asignatura o una parte de la geología. Los resultados obtenidos y la bibliografía consultada sugieren la necesidad de plantear nuevas investigaciones que den información sobre los diferentes objetos o cuerpos celestes representados en los dibujos y sobre las teorías alternativas de los estudiantes de las edades comentadas (14-18 años) acerca de la dinámica del Universo, en la medida en que para estos sectores educativos no se dispone, en líneas generales, de materiales publicados. En este sentido, no podrán modificarse las explicaciones alternativas que están teniendo los jóvenes de estas edades si no es conociéndolas con un mayor rigor; estas explicaciones incluyen conceptos físicos que se están dando por aprendidos y que la práctica docente constata como no ajustados al conocimiento científicamente aceptado. Por esta razón sería recomendable profundizar en esta línea reproduciendo, incluso, con las matizaciones necesarias, los trabajos que otros autores han desarrollado en enseñanza primaria básicamente (Nussbaun y Novak, 1976; Nussbaum, 1979; Klein, 1982; Sneider y Pulos, 1983 y Jones, Lynch y Reesink, 1987) para jôvenes de enseñanza secundaria y universitaria. 


\section{REFERENCIAS BIBLIOGRÁFICAS}

AUSUBEL, D.P., NOVAK, J.D. y HANESIAN, H. (1983). Psicología Educativa: un punto de vista educativo. Méjico: Trillas.

JONES, B.L., LYNCH, P.P. y REESINK, C. (1987). Children's conceptions of the Earth, Sun and Moon. International Journal of Science Education, 9 (1), pp. 43-54.

KLEIN, C.A. (1982). Children's concepts of the Earth and the Sun: A Cross Cultural Study. Science Education, $65(1)$, pp. 95-107.

MOREIRA, M.A. (1994). Cambio conceptual: crítica a modelos actuales y una proptesta a la luz de la teoría del aprendizaje significativo. II Simposio sobre Investigación en Educación en Física. Argentina.

NUSSBAUM, J. y NOVAK, J.D. (1976). An Assessment of
Children's Concepts of the Earth Utilizing Structured Interviews. Science Education, 60 (4), pp. 535-550.

NUSSBAUM, J. (1979). Children's Conceptions of the Earth as a Cosmic Body: A Cross Age Study. Science Education, 63 (1), pp. 83-93.

OSBORNE, R. y FREIBERG, P. (1990). El aprendizaje de las Ciencias: las implicaciones de la Ciencia Infantil. Madrid: Nascea.

RODRÍGUEZ PALMERO, G. y RODRÍGUEZ PALMERO, M.L. (1995). El Universo: La constnicción de un conocimiento. 5th. International Conference Teaching Astronomy. Vilanova i la Geltrú. Barcelona.

SNEIDER, C. y PULOS, S. (1983). Children's Cosmographies: Understanding the Earth's Shape and Gravity. Science Education, 63 (2), pp. 205-221. 
